\title{
DYNAMIC CONGESTION CONTROL IN WDM OPTICAL NETWORK
}

\author{
Sangita Samajpati ${ }^{1}$, Abhisek Daw ${ }^{2}$ and Swapan Kumar Mondal ${ }^{3}$ \\ ${ }^{1}$ Regent Education \& Research Foundation, Barrackpore, WB, India. \\ eva.samajpati@gmail.com \\ ${ }^{2}$ Tata Consultancy Services \\ abhisek. daw@tcs.com \\ ${ }^{3}$ Kalyani Govt.Engg.College, Kalyani, WB, India. \\ paltaswapan@yahoo.co.in
}

\begin{abstract}
This paper is based on Wavelength Division Multiplexing (WDM) optical networking. In this optical networking, prior to data transfer, lightpath establishment between source and destination nodes is usually carried out through a wavelength reservation protocol. This wavelength is reserved corresponding to a route between the source and destination and the route is chosen following any standard routing protocol based on shortest path. The backward reservation protocol is implemented initially. A fixed connected and weighted network is considered. The inputs of this implementation are the fixed network itself and its corresponding shortest path matrix. After this initial level of implementation, the average node usage over a time period is calculated and various thresholds for node usage are considered. Above threshold value, request arriving at that path selects its next shortest path. This concept is implemented on various wavelengths. The output represents the performance issues of dynamic congestion control.
\end{abstract}

\section{KEYWORDS}

Optical network, Wavelength Division Multiplexing, Backward reservation protocol, Congestion, Threshold.

\section{INTRODUCTION}

In recent years extensive research has taken place in the area of wavelength division multiplexing (WDM) networks. Wavelength-routed networks allow building high bandwidth networks. Optical networks employing wavelength division multiplexing (WDM) offer the promise of meeting the high bandwidth requirements of emerging communication applications. WDM technology has been recognized as one of the key components of the future networks. The commercialization of WDM technology is progressing rapidly. This work focuses on the dynamic control of fully optical wavelength routed WDM network, in other words how the arriving connections, i.e. lightpath requests, should be configured into the network in order to maximize the expected revenues [1].

A continuous path, having the same wavelength reserved in all the hops of the route, is called a lightpath. Since lightpaths are the basic building block of this network architecture, their effective establishment is crucial. It is thus important to provide routes to the lightpath requests and to assign wavelengths on each of the links along this route among the possible choices so as to

Rupak Bhattacharyya et al. (Eds) : ACER 2013, 
optimize a certain performance metric [2].The wavelengths assigned must be such that no two lightpaths that share a physical link use the same wavelength on that link. In distributed WDM optical networks having no wavelength conversion facility, usually a dedicated lightpath is first established between the source node (source) and destination node (destination), before the actual data transfer starts.

It is noted that with increase in Arrival Rate, blocking probability also increases. When more number of requests arrives in a specific time, more is the contention for free wavelength and more chance of getting blocked. Again with increase in the number of wavelength the blocking probability decreases as more wavelengths allow more requests to successfully select and reserve the selected wavelength. Thereby, successful data transmissions occur. But assigning more wavelength in a network increases the cost. Few protocols have been used for reducing the blocking probability. Among the various protocols, backward reservation protocol helps in reducing the chance of getting blocked to a significant extent. But with growing demand for wavelength due to increase in number of requests, backward reservation protocol also shows the increase in blocking probability. A brief description of existing backward reservation protocol with its problem has also been studied.

It has been observed in backward reservation protocol that when traffic fight for the selection of wavelength through their shortest path resulting in congestion when some nodes belong to the shortest path of a large number of requests, the wavelength available in the next shortest path remain unused [3]. With a good solution of this problem, more customers can be accommodated by the given system, and fewer customers need to be rejected during periods of congestion.

\section{OBJECTIVE}

Implement a protocol to dynamically reduce the congestion problem in wavelength assignment in WDM network and to maximize the resource utilization as well as minimize the cost. Also, the optimization goal is to minimize some infinite time horizon cost functions such as blocking probability.

\section{BACKWARD RESERVATION PROTOCOL (BRP)}

Lightpath establishment in a distributed environment requires a control protocol that will be responsible for a lightpath establishment and teardown facilities. These protocols can be categorized based on their working mechanisms and whether they require the use of global state information or not.

Parallel reservation scheme requires global state information in particular topology and wavelength usage information on each of the links in the network to work. A separate control message is sent from source node to each of the nodes in the path from source to destination for wavelength reservation. After a successful reservation of wavelengths by each node along the path it will send separate acknowledgement messages to the source node.

In BRP scheme control message (PROB) originated from the source node travels from source to destination on a hop by hop basis collecting wavelength usage on each node in the route. When this control message reaches at the destination node it selects a free wavelength from a set of free wavelengths along the entire path and then initiates a reservation message (RES) in the reverse direction from destination to source. This message reserves that particular wavelength on each node on the reverse path from destination to source [4]. However, it suffers from "outdated information". Fig. 1 shows the principle of BRP. 


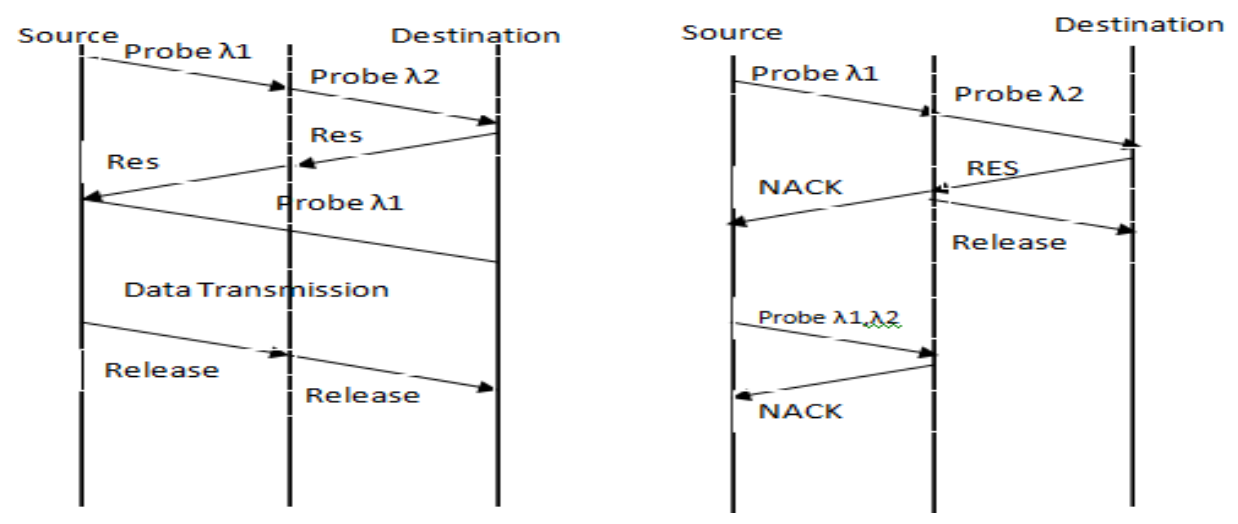

Fig. 1: Backward Reservation Protocol

\section{Congestion Control}

For every node pair, there may exist more than one feasible route and wavelength. An optimal algorithm should select a route and wavelength pair such that more requests can be accommodated into the network. Though this optimality criterion cannot be addressed directly, it is addressed in couple of indirect ways. Minimum amount of resources are used to establish the current request, so as to maximize the amount of free resources available in the network for future requests [5]. However, this may result in increasing the congestion in the network. In an alternate way of addressing this problem, algorithms try to balance the load in the network by selecting the next shortest path.

A network is said to be congested when the users of the network collectively demand more resources than the network has to offer. Congestion in a network leads to delayed packet arrivals, packet droppings and even denial of service for certain hosts [6].

\subsection{Congestion and the need to control it}

Ideally we want unlimited bandwidth for every user connected to the network. Reality however is different. We have limitations to the available bandwidth. Limitations that are not only because of the transport protocol but also of the medium for transferring informations (copper wires, electrical transmitters etc). Since the resources are limited and users and their demands are exponentially increasing we need to control the way network resources are used. We want to send as much traffic as possible through a link to maximize our throughput. On the other hand if we send at excessive rates then we will experience congestion. The network throughput will dramatically drop and we may even have a collapse. To avoid such situation congestion control is needed [7]. The need for new technology and network independent, congestion control algorithms is more demanding than ever. To satisfy these needs we must consider a new totally different approach.

\subsection{Working Principle of Dynamic Congestion Control}

The schemes considered under dynamic traffic can be divided into two cases reconfigurable and non-reconfigurable. If it is possible to reconfigure the whole network when blocking would occur, the blocking probability can be considerably reduced. Such an operation, however, interrupts all (or at least many) active lightpaths and requires a lot of coordination between all the nodes. 
In large networks the reconfiguration seems infeasible. In any case, the reconfiguration algorithm should try to minimize the number of reconfigured lightpaths in order to minimize the amount of interruptions in the service. The other case occurs when active lightpaths may not be reconfigured. In this case it is important to decide which route and wavelength are assigned to an arriving connection request in order to balance the load and minimize the future congestion in the network.

In general, one is interested in the optimal policy which maximizes or minimizes the expectation (infinite time horizon) of a given objective function. The objective is defined in terms of maximizing or minimizing some revenue or cost function. The cost may represent e.g. the loss of revenue due to blocked calls, where different revenue may be associated to each type of call.

\section{THRESHOLD IN NETWORK}

Based on average node usage, a value can be considered so that high usage of a node can be prevented. This value can be referred as threshold.

Congestion occurs when the traffic and resource mismatch at some points of the network. It is therefore usually local and changes rapidly. Changing routing table, which involves information exchange and route computation across the entire network, is often too costly and also too slow. When congestion does occur, the routing algorithm has to wait until next updating period to respond. At that time, the congestion may have already dissipated [8].And the reported high distances caused by the congestion, which are no longer valid, nevertheless has a misleading effect on the traffic that shares the same link when the next route updating is due.

As the traffic approaches the capacity of a path, it is often impossible for a single-path routing algorithm to derive satisfactory routes. It is necessary to provide some degree of load sharing by using multi-path routing.

Although most multi-path routing algorithms are too complex to be used in an adaptive fashion, some simple form of multi-path routing can be easily adopted and can still improve the performance. To make full use of the multi-equal-path routing with traffic-sensitive metrics, it may be necessary to transform the values of link distances into a set of discrete values. When the traffic is heavy and changing rapidly, it is difficult for the routing algorithm to determine the entire routes based on the past link distance information. It has to allow continuous changes in routing decisions. When the traffic accumulated in some areas of the network exceeds a threshold, an alternate route can be provided to ease the congestion [5].

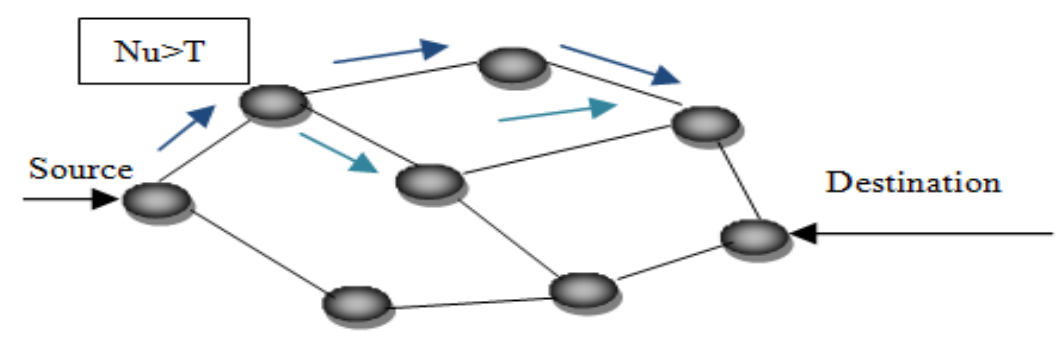

Fig. 2: Selection of new route due to congestion

In network, the available shortest path selection algorithms were proposed and evaluated. The shortest path selects the shortest hop path among the all paths [4]. It is important to note that though every link on a path computed has free wavelengths, a connection may still get blocked 
due to unavailability of a single continuous free wavelength. The available shortest path routing algorithm considers wavelength availability while computing a route. When a request arrives in a particular node, it compares the node's wavelength usage with a given threshold value \& if the usage is above of threshold then it chooses the next shortest path by the shortest path algorithm. In this work not only considered wavelength availability but also link congestion information to dynamically compute a route. Fig. 2 shows that a request has to change its path as one of the nodes' usages is greater than the threshold value in which " $\mathrm{Nu}$ " represents the node's usages and "T" represents threshold value.

\section{Simulation OF RESUltS}

In order to evaluate the proposed protocol with different thresholds and compare it with backward reservation protocol (BRP) varying we had to implement the proper test environment. In this section we see how that was accomplished and then comment on the results that were gathered from our simulations.

\subsection{Implementation}

In order to simulate the proposed protocol the following steps are considered.

Step - 1: Generation of shortest path: Implementation of the input to Floyd's algorithm with random fit approach and obtaining the output of Floyd's algorithm.

- Input: Numbers of the nodes in the graph and percentage of connectedness in the graph.

- Output: Randomly generated connected, weighted network, all pairs shortest distance matrix and path matrix.

Step - 2: Generation of requests: Generation of Requests with a mean arrival rate, randomly generated source and destination pairs. Queuing of the requests is done.

Step - 3: Probing: A source sends a PROB packet to its destination to gather information about wavelength usage without locking any wavelength. The PROB packet carries a bit vector to represent the set of wavelength available to establish the connection.

Step - 4: Decision based on Average node's usage: After arriving at each node in the designated path, the request checks the average usage of the node.

Step - 5: Compare with threshold: If the average usage is above threshold value, the next shortest path for that request is calculated by Floyd Algorithm and execution again begins from (3), else it proceeds through that path.

Step - 6: Reservation: The destination picks up one of the wavelengths randomly from the pool of available wavelengths and reserves the selected wavelength by traversing in the reverse order.

Step - 7: Unavailability of wavelength: In case of unavailability of wavelength during reservation, the request is blocked.

Step - 8: Data Transmission: After successful reservation, data transmission takes place completely.

Step - 9: Simulation: Simulation of the relation of blocking probability of connection request with mean arrival rate of request, wavelength and threshold of node's usage in a network. 


\section{SiMUlation RESUltS AND DiSCUSSION}

Simulation of the relation of blocking probability of connection request for varying mean arrival rate of request with different thresholds for different wavelengths, varying thresholds (TH) with various mean arrival rate and for number of wavelengths per channel using random fit approach in a network. Arrival of connection request follows poison arrival process. In this simulation the input is the shortest distance matrix and shortest path matrix (obtained following Floyd's algorithm) corresponding to the fixed network.

\subsection{Analysis Based on Arrival Rate (AR):}

In Fig. $3-5$, the blocking probability of connection request for varying mean arrival rate of request with different thresholds is shown. The x-axis represents the arrival rate of a connection request and the y-axis represents the blocking probability of the request.

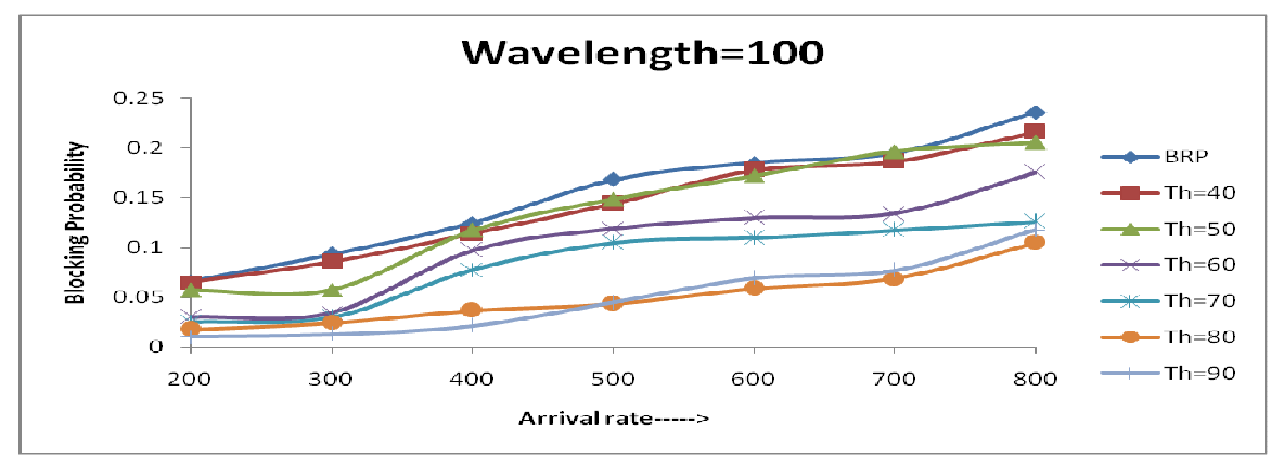

Fig. 3: Blocking Probability vs. Arrival Rate with wavelength $=100$

The Fig. 3 shows the performance of the proposed protocol with respect to backward reservation protocol .The number of wavelength channel is set to 100. Threshold 40, 50 does not show better result than brp as when threshold is too low most of the requests are forced to change their paths and they in turn increase the nodes' usage in the changed path. The graph shows two natures. One is upto arrival rate 500 and another above arrival rate 500.So it is better to study them separately.

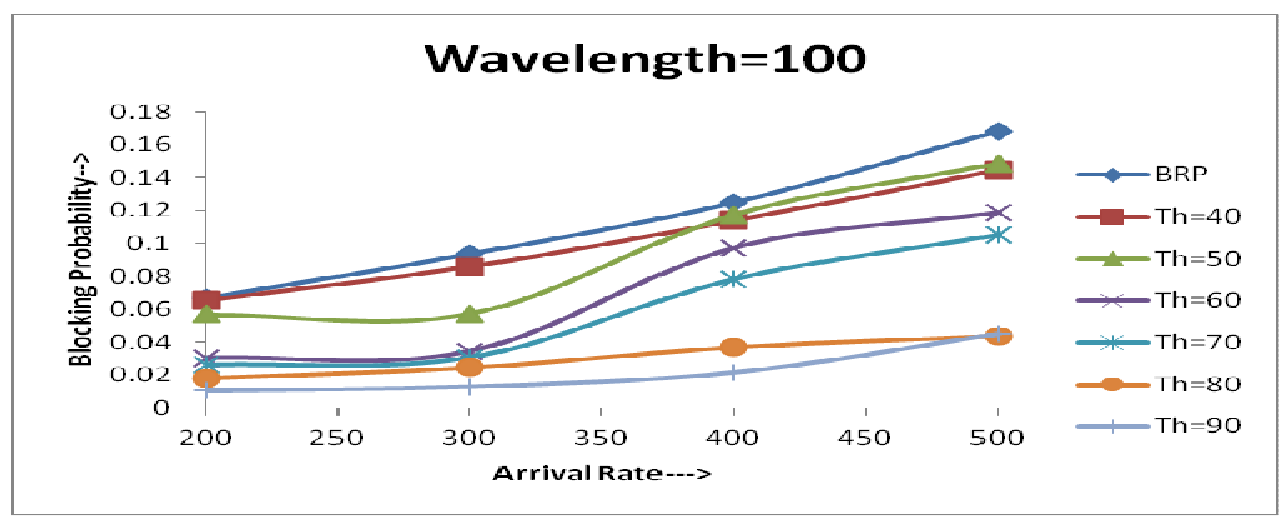

Fig. 3a: Blocking Probability vs Arrival Rate upto AR $=500$ 


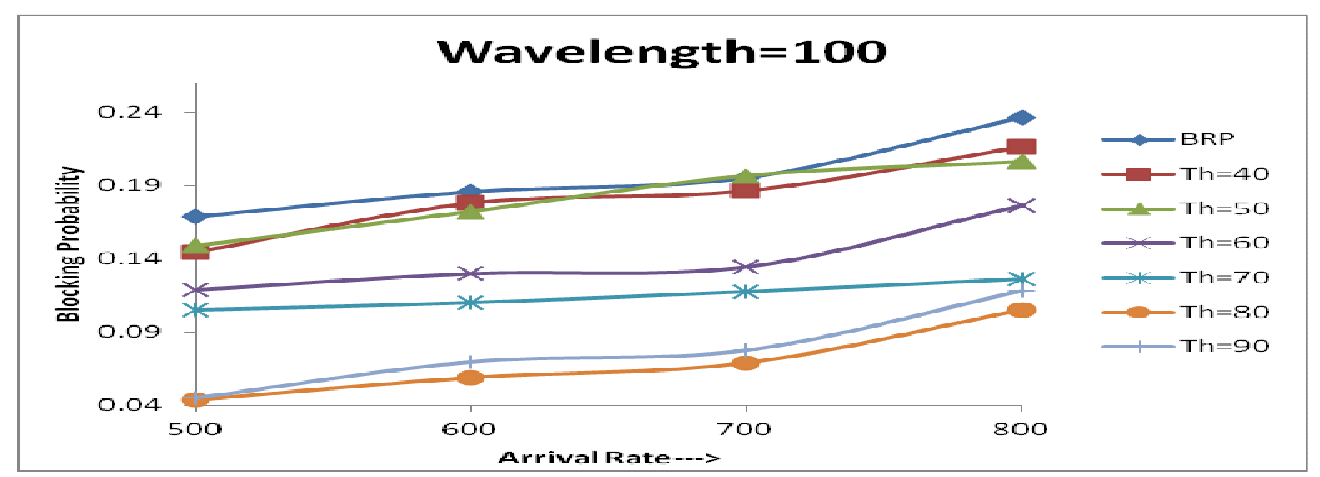

Fig. 3b: Blocking Probability vs Arrival Rate above AR = 500

Thresholds 80, 90 shows better result here as most of the requests are allowed to go through the original path. From Fig. 3a it can be seen that till arrival rate 500, threshold 90 shows better result. While from Fig. 3b, it is seen that above AR 500 threshold 80 is better as in case of 90, the chance of blocking increases during reservation. In threshold 80 , however as comparatively less number of requests is allowed to probe through original path, less chance of getting blocked during reservation.

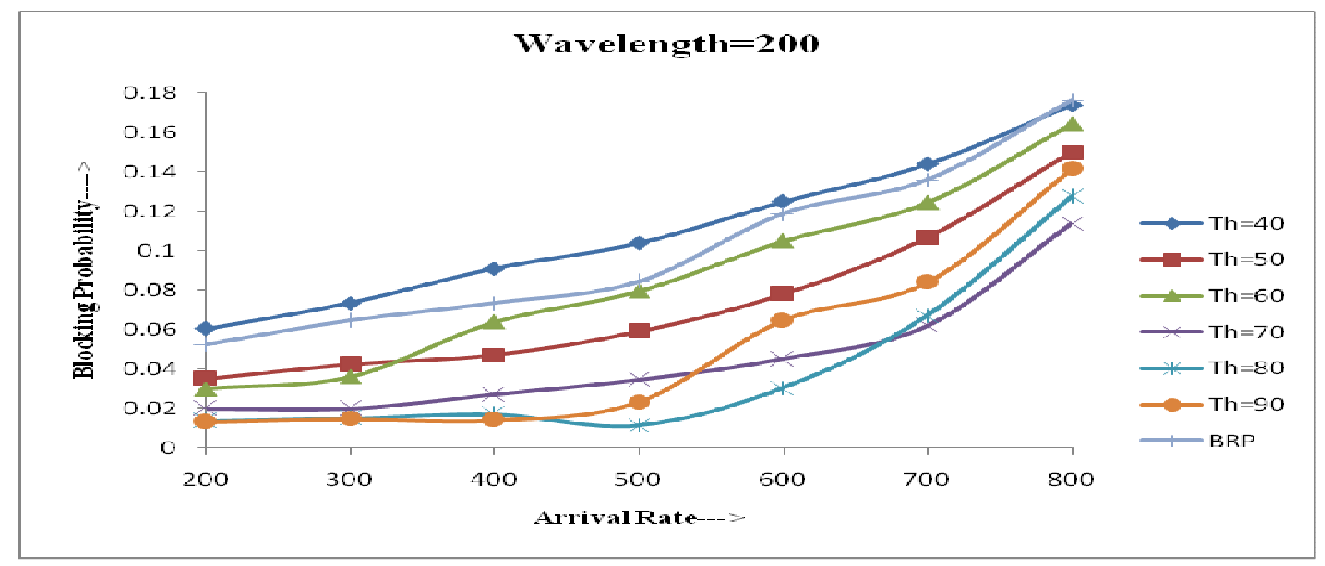

Fig. 4: Blocking Probability vs. Arrival Rate with Wavelength $=200$

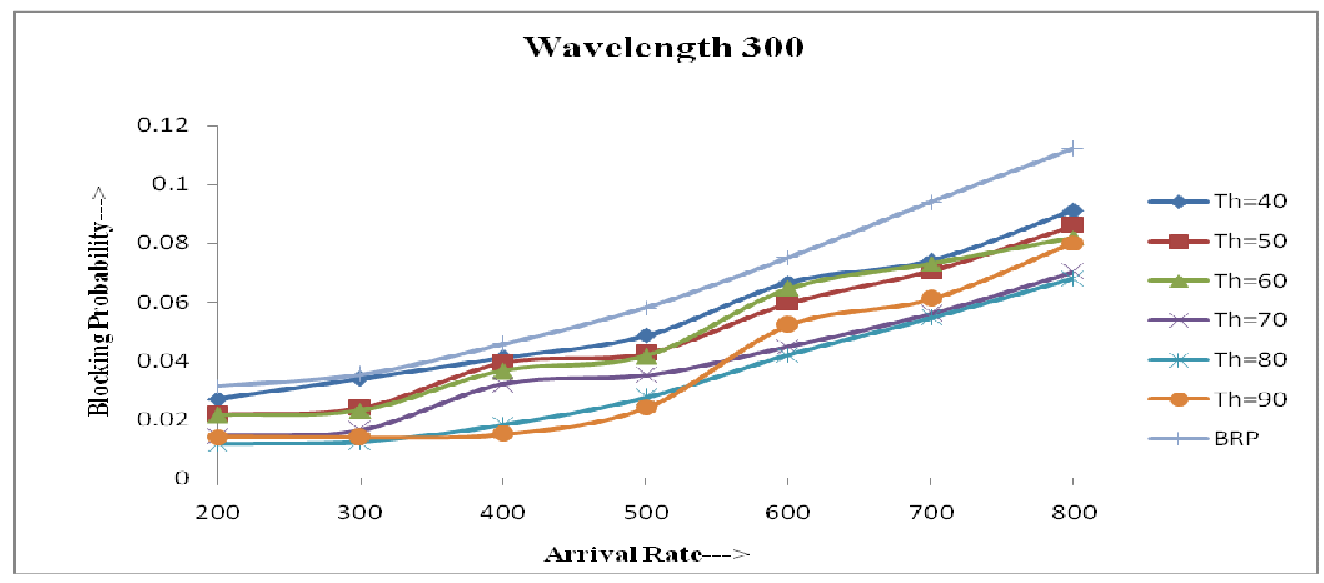

Fig. 5: Blocking Probability vs. Arrival Rate with wavelength $=300$ 
Fig. 4 \& Fig. 5 show the blocking probability when wavelength is 200 and 300 respectively with varying different thresholds. Low threshold 40 does not show better result than BRP as when threshold is too low most of the requests are forced to change their path and they in turn increase the nodes' usage in the changed path. Higher thresholds 80,90 shows better result here as most of the requests are allowed to go through the original path. Till arrival rate 400, threshold 90 shows better result .above AR 500 threshold 70-80 are better as in case of 90, the chance of blocking increases during reservation. In threshold 70-80, however as comparatively less number of requests is allowed to probe through original path, less chance of getting blocked during reservation.

\subsection{Analysis Based on Wavelength:}

In case of analysis based on wavelength we have studied the performance of wavelengths with respect to brp varying different thresholds with a fixed arrival rate 300 i.e. high arrival rate.

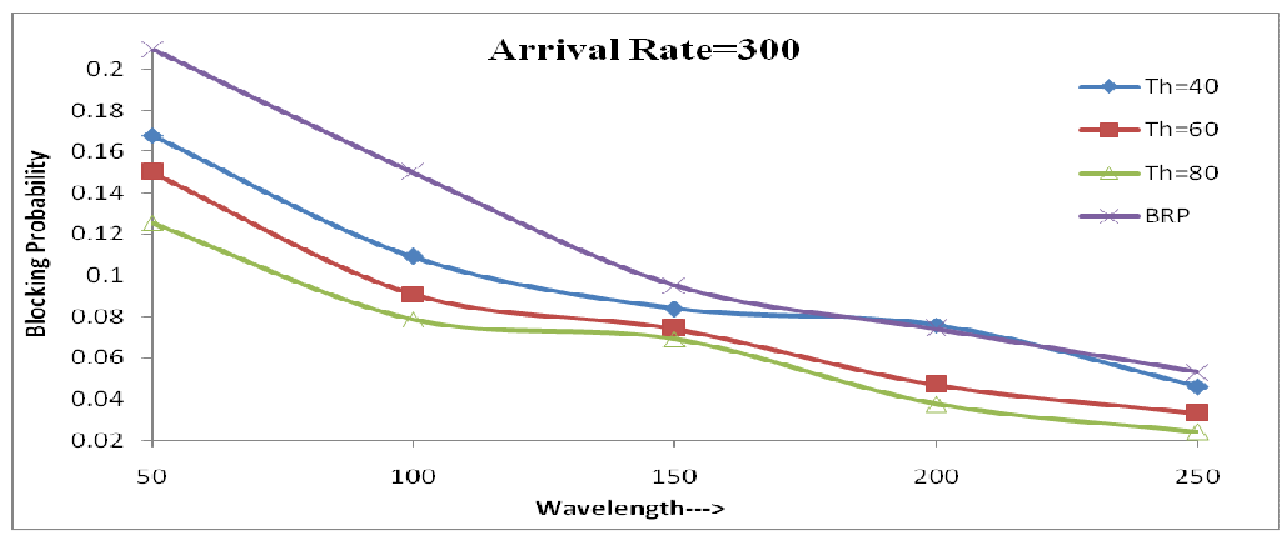

Fig. 6: Blocking Probability vs. Wavelength with mean Arrival Rate $=300$

We can see in Fig. 6 in where threshold 80 clearly shows better result than other thresholds as pointed in other curves. With increase in wavelength, brp decreases as in normal case .

\subsection{Analysis Based on Threshold:}

The graphical analysis based on thresholds shows the blocking probability of a connection request with respect to thresholds varying different wavelengths.

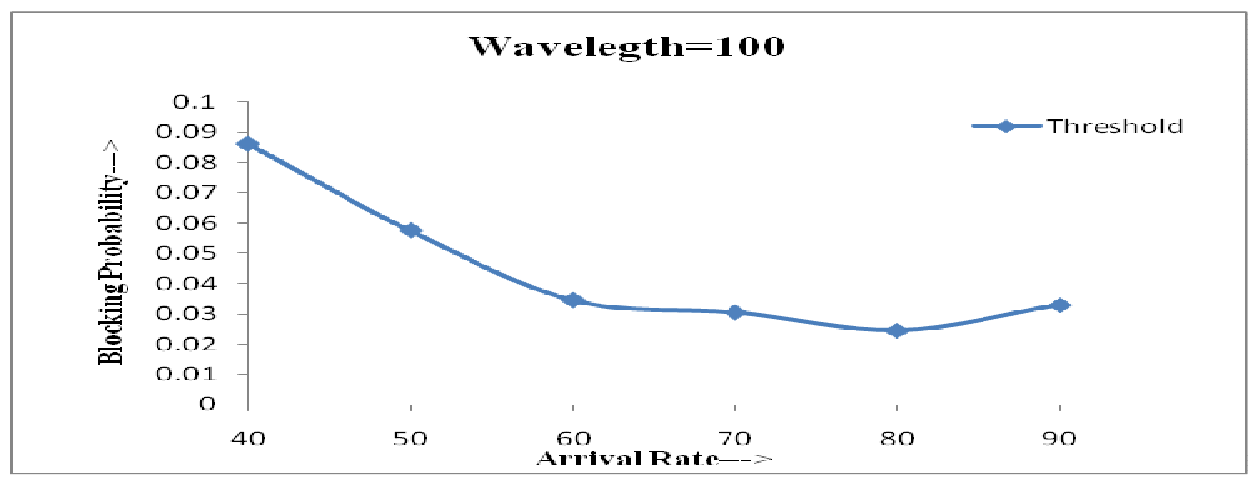

Fig. 7: Blocking Probability vs. Arrival Rate with Wavelength $=100$ 


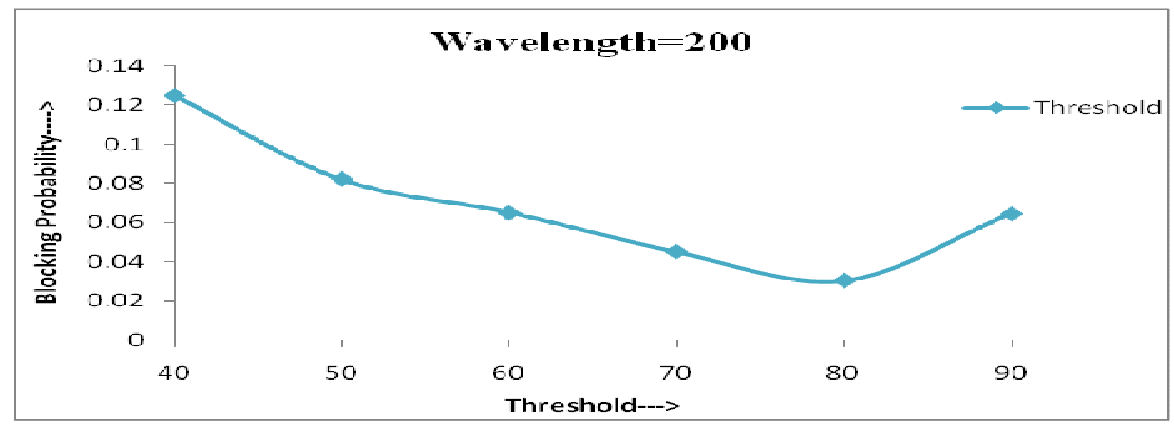

Fig. 8: Blocking Probability vs. Thresholds with Wavelength $=200$

From Fig. 7 and Fig. 8 it can be conferred that when the threshold increases the blocking probability decreases. But after a certain point the Blocking probability increases. Threshold 80 shows better result than other thresholds.

The requests follow the path obtained by using shortest path routing protocol. Some nodes may be used frequently as they belong to shortest paths of many requests. As a result the link usages of those nodes are very high. So, the pool of free wavelengths for that link gets depleted and resulting in blocking of few requests. But those requests can use other sub-optimal paths and successful data transmission can occur.

The threshold value needs to be determined accurately. As, some inaccurate selection of threshold like in case of low threshold values, might result in chaotic and abnormal situation as requests, being forced to select next shortest path, nodes belonging to next shortest path might be in some request's optimal path and hence increases that node's usage. So, it has been found from analysis of all the results that using a high threshold value in the range of 70-90 one can achieve a better result

Again a situation might arise when the inter-connections are very low. So, many sub-optimal shortest paths may not exist as a result requests get blocked. Selection of a threshold value is very important.

\section{Conclusions}

In this work, however, we have considered only the wavelength routed networks. Nonetheless, the promising future of WDM networks makes them an interesting topic for the research. The objective was to search for a way to reduce the node congestion in a heavy busty traffic in the area of optical networking. Because of the large amount of activity on this subject over the past dozen years, capturing the important and most significant developments in a concise format is a challenging task; thus, apologize if we inadvertently left out some contributions which could be considered to be more important. We have introduced a backward reservation protocol and implemented a solution to control the congestion in WDM network by lowering the blocking probability. This is accomplished by allowing the nodes that are considered to be the main decision points for selecting the shortest path and also try another shortest path if the first choice does not succeed.

It has been observed that for comparatively low arrival rates threshold 80 or 90 gives better result. But as the arrival rate is increased it has been observed that threshold 70 or 80 provide better result compare to 80 or 90 . 
More study can be done on the effect of other parameters like use of control packets, setup time, time period etc. Piggybacking of intermittent control packets with link usage information for updating the network to reduce the effect of outdated information of wavelength usage in the network can also be implemented. Results can be made more statistically stable. Concept of threshold can be tested in other protocols also. The running time of the algorithm can also be reduced.

\section{REFERENCES}

[1] Zang, H., Jue, J., Sahasrabuddhe, L., Ramamurthy, R., \& Mukherjee, B., (2001) "Dynamic lightpath establishment in wavelength-routed WDM networks", IEEE Communication Magazine, Vol. 39, No. 9, pp. 100-108.

[2] Ozdaglar, Asuman E., \& Bertsekas, Dimitri P., (2003) "Routing and Wavelength Assignment in Optical Networks", IEEE/ACM Transactions on Networking (TON), Vol. 11, No. 2, pp. 259-272.

[3] Todimala, A., \& Ramamurthy, B., (2003) "Congestion-based Algorithms for Online Routing in Optical WDM Mesh Networks", Proceedings of Second IASTED International Conference on Communications, Internet, and Information Technology.

[4] Zhang, H. \& Mukherjee, B., (2003) "Lectures on Routing and wavelength assignments for Wavelength-routed WDM Networks", Internetworking Technologies Handbook, Vol. 2, pp. 1 - 20.

[5] Thodime, G.P.V., Vokkarane, V. M., \& Jue, J. P., (2003) "Dynamic congestion-based load balanced routing in optical burst-switched networks", In GLOBECOM 2003 - IEEE Global Telecommunications Conference, pp. 2628-2632.

[6] Jonnadula,Venkata R., (2005) "Performance Analysis of Congestion Control Schemes in OBS Mesh Networks".

[7] Rossides, L., (1999) "Congestion Control in Integrated Services Networks", Masters Thesis, Dept of Computer Science, University of Cyprus, pp. 1-64.

[8] Wang, Z., \& Crowcroft, J., (1992) "Analysis of Shortest-Path Routing Algorithms in a Dynamic Network Environment”, ACM SIGCOMM Computer Communication Review, Vol. 22, Issue 2, pp. 63-71.

\section{AUTHORS}

Sangita Samajpati received his B.Tech. and M.Tech. degrees in Computer Science Engineering from the Kalyani Govt. Engineering College, Kalyani, West Bengal, India. She is currently an Assistant Professor at Regent Education \& Research Foundation. Her research interests include Optical Network, Fuzzy Logic and soft computing Technique.

Abhisek Daw received his B.Tech. and M.Tech. degrees in Computer Science Engineering from Institute of Technology and Marine Engineering, West Bengal, India and Kalyani Govt. Engineering College, Kalyani, West Bengal, India respectively. He is currently employed as an Assistant System Engineer in Tata Consultancy Services. His research interests include Optical Network, Fuzzy Logic and soft computing Technique.

Dr. Swapan Kr. Mondal received his Ph.D. degree from Jadavpur University, West Bengal, India. He did B.E. and M.Tech. degrees in Electrical Engineering and Computer Science Engineering from Jalpaiguri Government Engineering College, West Bengal, India and Rajabazar Science College, University of Calcutta, West Bengal, India respectively. Also he has M.B.A. in portfolio from IGNOU. Currently he is a Professor at Kalyani Govt. Engineering College, Kalyani, West Bengal, India and he has more than 23 years of teaching experience. He has some research publications in different reputed

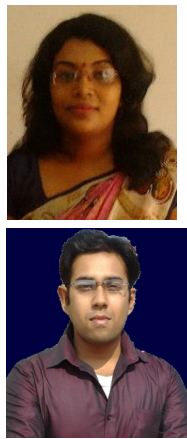
Journals and Conferences. His research interests include Computer Networking, Optical Networking and Computer Architecture. 